\title{
Interval type 2 fuzzy localization for wireless sensor networks
}

\author{
Noura Baccar ${ }^{1 *}$ and Ridha Bouallegue ${ }^{2}$
}

\begin{abstract}
Indoor localization in wireless sensor networks (WSN) is a challenging process. This paper proposes a new approach to solve the localization problematic. A fuzzy linguistic localization scheme is proposed. Based on interval type 2 fuzzy logic (IT2FL), a signal processing of the radio signal strength indicator (RSSI) minimizes the uncertainty in RSSI measurements from anchors caused by the indoor obstacles. The fuzzy system subdivides the map on fuzzy sets described by a new fuzzy location indicator (FLI). Fluctuations on RSS fingerprints are then reduced thanks to the IT2FL in the input side and the FLI in the output side. Experimentations were done in the Cynapsys indoor environment on a WSN test bed. The experimental results prove higher success rate in position estimations thanks to the FLI concept and the superiority of interval type 2 fuzzy logic to handle signal fluctuations.
\end{abstract}

Keywords: Interval type 2 fuzzy logic, Signal processing, Wireless sensor network, Localization

\section{Introduction}

Do we really need $x, y$, and $z$ coordination for indoor localization? When we are subjected to human localization process, we refer generally to linguistic localization (near to the desk, next to the window in front of the TV...). Thus, a fuzzification of this problematic will change our angle of view and enlarge our perception of localization methods from Euclidean geometrical equations and signal propagation models to more opened intelligent and pervasive computing.

The availability and diversity of wireless communication (wireless area networks (WAN), wireless sensor networks (WSN)...) give researchers a huge amount of creative areas. Those available transmitted data and indicators may be exploited in applications to facilitate human being life. Smart buildings, smart homes, and ubiquitous cities are the trends of leading projects in pioneer companies. Hence, the progress in WSN deployment for "smart" purposes, besides the implication of those huge technological companies in this field, give a big motivation to innovate in various communication techniques. Mobility and localization are indeed two constraining factors relative to WSN design problematic. Many emerging context-aware applications are stand on location-based services (LBS).

\footnotetext{
* Correspondence: baccar_noura@hotmail.com

${ }^{1}$ Innov'COM/ENIT/Cynapsys, University Tunis El Manar, Tunis, Tunisia Full list of author information is available at the end of the article
}

Since that, geo-localization in WSN has been the subject of many researches. For outdoor as well as indoor environment, the use of computational intelligence in localization techniques is not a new invented methodology. However, the specific nature of the indoor environment (shadowing, reflection, path loss...) originates depth investigation using different optimization techniques. Type 1 fuzzy logic (T1FL) is one of those techniques for geometrical localization and as a clustering-based methodology. Nevertheless, there has not been any attempt to investigate the usage of interval type 2 fuzzy logic (IT2FL) in indoor geo-localization. But, it was proved that the use of IT2FL in complex real-word applications presenting a high level of uncertainty in measurements performs better [1-3]. In control theory, some industrial application results were presented in [4], and the type 2 fuzzy logic controller (FLC) was applied to three domains: industrial control, mobile robot control, and ambient intelligent environment control. The author proved that type 2 FLC for each application provides smooth responses outperforming always the type 1 counterparts. This is due to the powerful paradigm of type 2 FLC to handle the high level of uncertainties present in real-world environments.

In [5], a data-driven IT2 fuzzy logic modeling framework is presented and very good computational efficiency was demonstrated through real industrial case study posing particular challenges in terms of data uncertainty comparing 
to type 1 fuzzy logic. The superiority of IT2FL to type 1 in handling measurement uncertainties in real-world applications was also proved in [6] and [7].

Since the localization problematic that we discuss here is based on radio signal strength indicator which is submitted to a high level of fluctuations and uncertainty in indoor environments and Interval Type 2 Fuzzy Localization System (IT2FLS) was proved to give better results dealing with data uncertainty, the use of IT2FLS may give similar results on the radio frequency (RF)based localization.

In this paper, a new approach on fuzzy geo-localization is proposed. Based on a linguistic concept, the expert builds an adaptive fuzzy model to the target environment. In a first learning stage, he defines the distribution of anchor nodes in a manner to cover all target space. Then, he ranges the radio signal strength indicator (RSSI) using linguistic fuzzy descriptors \{low, medium, or high\}. Because of the instability in the indoor RSSI measurements, an IT2FL processing is programmed. On the other hand, the expert clustered the target map on fuzzy sets using a new fuzzy location indicator (FLI). Thus, for each FLI, the expert takes the RSS fingerprints and proceeds to rule base building. Through semantic relations, the geometrical map dispositions are fuzzified to an "if-then" linguistic description. In the online stage, signals are submitted to IT2 fuzzification and then aggregated using the inference engine of the fuzzy localization system (FLS). The FLI is defuzzified to a crisp value describing the location zone in the map. Experiments in the Cynapsys indoor environment have proved the effectiveness of this approach.

This paper is organized as follows. Section 2 will present the background of localization algorithm based on fuzzy logic. Section 3 will detail the proposed approach concepts. In Section 4, the experimentation process and the results are discussed. Finally, the paper is summarized by a conclusion and perspectives.

\section{Background}

Two main technological choices are basic for the design of localization systems: the localization technology and the positioning technique. Firstly, location-based systems are generally RF-based technologies. Thanks to the speedy progress of nanotechnology [8] (the ease to reach receivers and sensors) that heavily involves short-range communications notably WiFi, Bluetooth, and ZigBee, localization applications become available and some are based on hybrid systems, from robotic guiding [9] to location-based services. In their survey [10], Liu et al. present existent indoor applications in the market and their different performance criteria. They concluded that fingerprinting schemes are better on indoor open areas.

Secondly, the localization techniques can be classified in three categories. The first one consists of deterministic techniques, classified as geometrical methods. They are range-based and estimate the target coordination through multi-lateration, triangulation, angulation, angle of arrival (AoA), and time of arrival (TOA) needing most of the time specific hardware. In their work, Yan et al. [11] presented a fuzzy-based geometrical probabilistic method to deal with non-light-of-sight (NLOS) conditions. Although it presents good results, their algorithm needs complex calculations and depends on the known and precision of anchor coordination.

On the other side, a big number of research works consider the probabilistic approaches [10] like Bayesian algorithms [12] and a third localization process is based on machine learning approaches [13-15], using SVM [16] and neural network-based algorithms [17]. In this category, K-nearest neighbor (KNN) classification was deeply investigated in fingerprinting algorithms $[14,18,19]$. It shows promoting results in terms of offering adequate estimation accuracy; however, a big number of anchors are required to reach this accuracy.

Fuzzy logic was exploited in two manners: geometric fuzzification concept [20] and rule-based fuzzification concept. Wang et al. [20] demonstrate that the fuzzy geometric approach outperforms the traditional least squares approach. However, this approach is costly while it requires velocity and azimuth angle measurements. Besides, the system is only adaptable to linear trajectories and not for the various kinds of fuzzy observer trajectories. Fan et al. [21] suggest the use of fuzzy logic in a recursive least squares filter. Although it proposes a different methodology to process noise unlike statistical models, it uses the classical coordinate-based localization technique in a way raising the filtering processes leading to the increase of computational complexity.

Garcia-Valverde et al. in [22] and [23] have worked on a mobile application based on fuzzy logic. They build an adaptive rule-based model to the system. Through a T1 fuzzification in RSS classification, the used technique is able to automatically learn offline and online to adapt in order to deal with the environmental changes. It reaches $82.22 \%$ of success accuracy. But to handle RSS fluctuations, they used two alternatives: a heuristic preprocessing algorithm and a responsive universal based on trim-like operation to remove peaks and drops and on a modified standard deviation-based technique applied to the last received RSSI values for every access point. On the other side, paper [24] investigates the RSS-based range-free fuzzy ring method. This approach proves good performance face to radio propagation irregularity. However, it is computational intensive and difficult in experimental deployments while it depends on the propagation model parameter estimation.

In [25], the author uses T1FL for RSS clustering and the FLS creates linear equations to estimate the location 
zone. This system provides $95 \%$ accuracy in positioning, whereas it has big granularity in localization (zones and not rooms) which are relatively away from each other and without providing results in case of adjacent rooms.

Furthermore, IT2FL was not used for localization. It is used generally in WSN for clustering sensed data. Although Liang and Wang in [26] presented a methodology to simulate uncertainty on RSSi and to cluster measurements, it was not exploited on localization. In literature, no real experiments on indoor mobile application based on type 2 fuzzy logic were found and this is the main contribution of this paper.

Our anchor-based proposed approach provides high granularity in location definition. It uses no preprocessing algorithm but the IT2 in the fuzzification phase which will handle RSS fluctuations. The following section will give all details of the proposed approach.

\section{Proposed approach}

Generally, when working on geo-localization, geometric positioning is used to calculate the coordinates of mobile nodes. Little work referred to "linguistic" geo-localization. In our proposed linguistic approach, calculation of the mobile's node position is based on hierarchical fuzzy clustering process. As presented in Fig. 1, in the first learning phase, the target space is subdivided in zones characterized by a FLI. The FLI is incremented in order to respect continuity in the geometric space and translation between rooms. This continuity in FLI will guarantee the continuity in fuzzy space where each room is considered as a fuzzy set (FS). Thus, each room is described by a fuzzy vector of FLIn.

In each FLI, RSS measurements are collected from anchor nodes in the data base (DB). Those measurements are processed using interval type 2 fuzzy logic algorithm to minimize instability of this indicator through its footprint of uncertainty (FOU) property. Based on the created $\mathrm{DB}$, the expert extracts the linguistic rules to form the rule base of the fuzzy process.

On the online phase, RSSi measurements are first fuzzified using IT2 process as described in Fig. 2 and transmitted to the inference engine which will proceed to implication and aggregation methods referring to the rule base. The aggregated type 2 output will be then type-reduced to a fuzzy type 1 FLI. Finally, the defuzzification module will conclude the location estimation process by calculating the crisp corresponding FLI.

\subsection{Fuzzy interval type $\mathbf{2}$ input processing}

An intelligent localization algorithm is proposed based on "interval type 2 fuzzy logic" for input processing. Type 2 FLS is generally used when the circumstances are too unknown to determine exact membership grade like when the training data is affected by noise. In a big number of control and clustering applications, higher accuracy has been proved using the IT2 fuzzy logic [27]. Satvir et al.

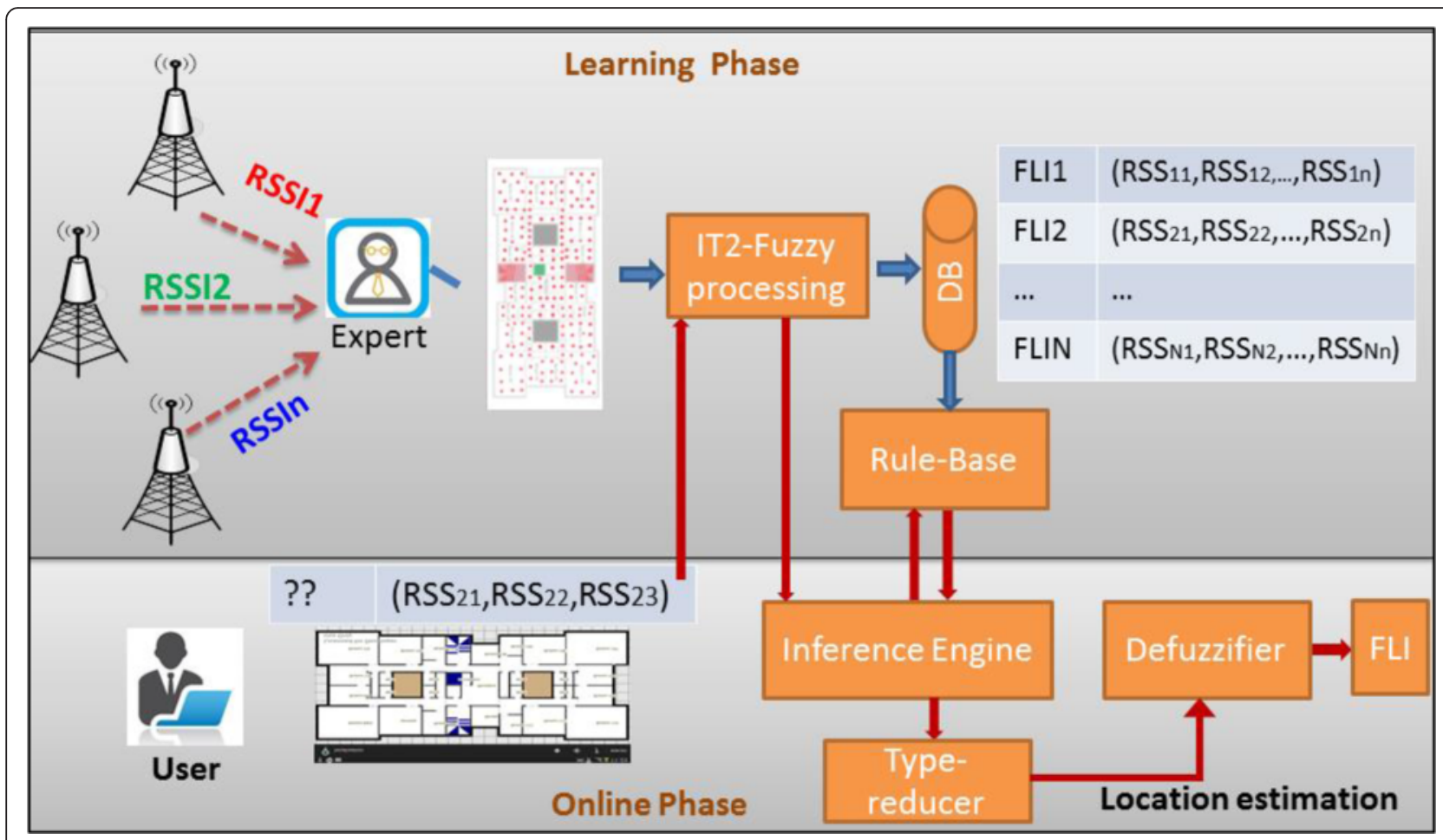

Fig. 1 General fuzzy localization approach diagram 


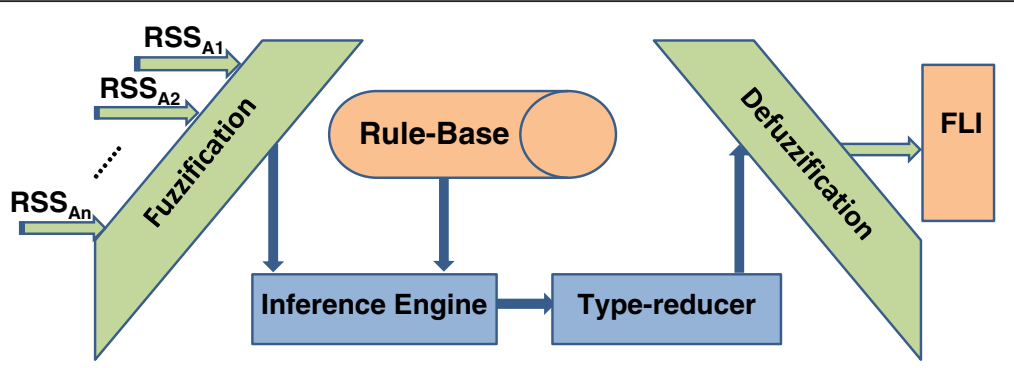

Fig. 2 Interval type 2 fuzzy process

[27] proved the viability of interval type 2 over type 1 FLSs through implemented systems in real environment. IT2 handled the presented noise by its uncertainty modeling property.

In their work [28], Aladi et al. demonstrated the relationship between the FOU size and the amount of uncertainty and noise in a given environmental setting. Thus, considering the target space as fuzzy sets will incorporate fluctuations in RSS measurements. An RSS vector in a zone will not be as specific as it was saved in the learning phase. The fuzzification process will limit this specificity and takes into consideration the instability of signal propagation in the indoor environment.

Two approaches exist to design an IT2FLS: the first approach is partially dependent and based on a type 1 FLS design and then a translation to the IT2FLS. Thus, a faster comparative study between $\mathrm{T} 1$ and the IT2 could be easily done. The second approach relies on a direct design of predefined IT2FLS parameters and thus avoids the effect of translation from T1 which may not give the best results.

As in this work, we intend to compare the results of the T1 and IT2 in localization error, and to prove the superiority of IT2 upon T1, the first approach is used. Thus, we will preserve the basic structure (the number of membership functions and the rule base).

Zadeh defines "Fuzzy Logic is determined as a set of mathematical principles for knowledge representation based on degrees of membership rather than on crisp membership of classical binary logic." RSS is T1 FS representing RSSi $(\mathrm{t})$ in the fuzzy domain. Based on the Zadeh theory, it can be defined as

$$
\mathrm{RSS}=\int_{D_{R S S}} \mu_{R S S}(x) / x
$$

where $D_{\mathrm{RSS}}$ is the universe of discourse: $D_{\mathrm{RSS}}=[-100,-70]$; $\mu_{\mathrm{RSS}}$ is the membership function (MF): $\mu_{\mathrm{RSS}}: D_{\mathrm{RSS}} \rightarrow[0,1]$; and " $\int$ "denotes the collection of all points $x \in D_{\text {RSS }}$ with associated membership grade $\mu_{\mathrm{RSS}}(x)$

Consider an IT2FS $\widetilde{R S S}$ described based on the definition in [29]:

$$
\begin{aligned}
\widetilde{\mathrm{RSS}} & =\int_{x \in D_{\mathrm{RSS}}} \int_{u \in J_{x \subseteq[0,1]}} \mu \widetilde{\mathrm{RSS}(x, u)}(x, u) \\
& =\int_{x \in D_{\mathrm{RSS}}}\left[\int_{u \in J_{x \leq[0,1]}} 1 / u\right] / x
\end{aligned}
$$

where $x$ is the primary variable in $\widetilde{D_{\mathrm{RSS}}}=[-100,-70]$; $u \in[0,1]$ the secondary variable in the domain $J_{x \subseteq[0,1]}$ at each $x \in \widetilde{\mathrm{D}_{\mathrm{RSS}}}$; and the amplitude of $\mu_{\widetilde{R S S}(x, u)}$ is the secondary grade of $\widetilde{\mathrm{RSS}}$, equals 1 for $\forall \mathrm{x} \in \widetilde{\mathrm{D}_{\mathrm{RSS}}}$ and $\forall$ $u \in J_{x} \subseteq[0,1]$.

$\widetilde{\mathrm{RSS}}$ is described by its FOU, shown in the right part of Fig. 3 using the upper and the lower membership functions $\mu \overline{\mathrm{RSS}}$ and $\mu_{\mathrm{RSS}}$ [29] as
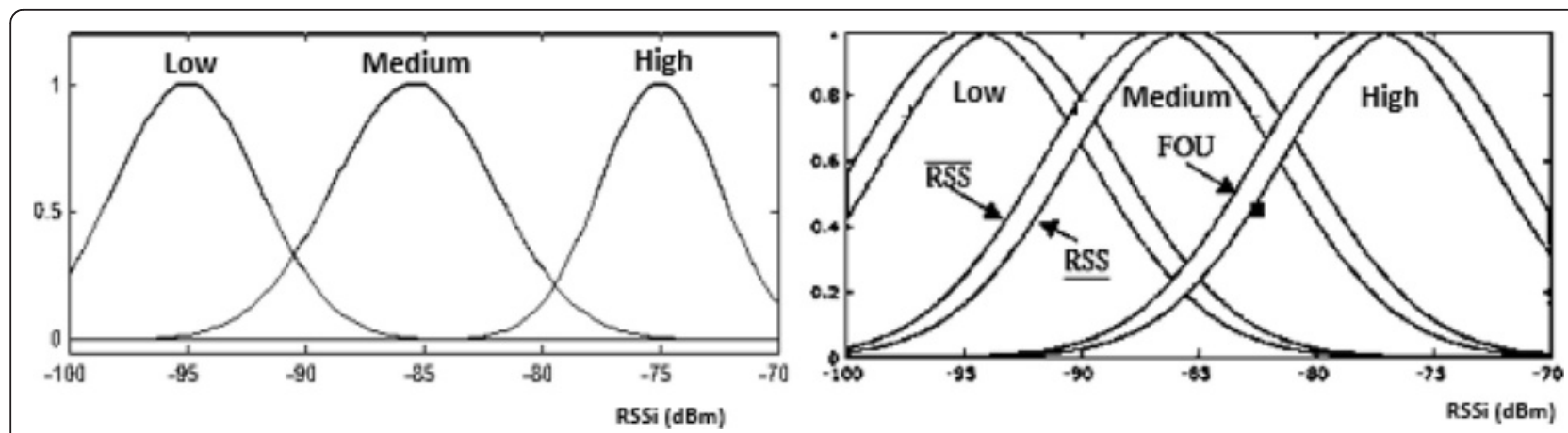

Fig. 3 Type 1 (left) and interval type 2 (right) RSS fuzzy sets (dBm) 


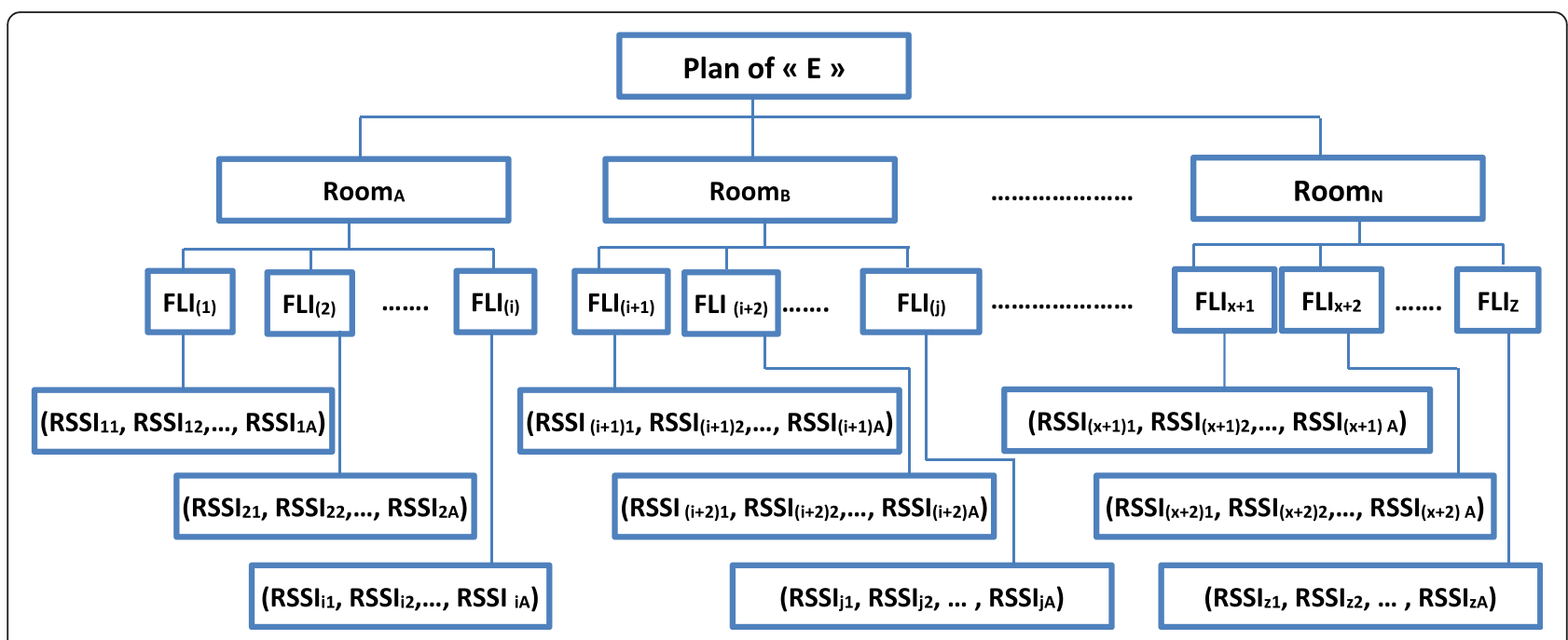

Fig. 4 Fuzzy location indicator (FLI) diagram

$$
\mathrm{FOU}(\widetilde{\mathrm{RSS}})=\cup_{\forall x \in J_{x}}\left(\mu_{\overline{\mathrm{RSS}}}, \underline{\mu_{\mathrm{RSS}}}\right)
$$

The FOU is not uniform in the FS's region. With an assumption that the noise/uncertainty is uniform, a FOU construction method gave rise to an equal amount of uncertainty in the memberships.

In order to ensure a uniform FOU, based on the work of Aladi et al. [28] considering the FOU size, it is expressed using the parameter $c \in[0,1]$. For $c=0$, MFs are of type $1 \mathrm{FS}$, and for $c=1$, they are an IT2 set with a very wide FOU. Thus, the upper and lower MFs are expressed as follows:

$$
\begin{aligned}
\mu_{\mathrm{RSS}} & =\min \left[\mu_{R S S}(x)+\frac{c}{2}, 1\right] \\
\mu_{\underline{\mathrm{RSS}}} & =\min \left[\max \left[\mu_{R S S}(x)-\frac{c}{2}, 0\right], 1-c\right]
\end{aligned}
$$

Gaussian membership functions are considered in both T1 and IT2FLS.

\subsection{Output processing: the FLI}

Through a linearization process of the 2D plan, the tagged environment "E" will be hierarchized in $\mathrm{N}$ fuzzy sets, Z fuzzy subsets (FLI).
To be localized, a mobile node sends a message to the nearest anchor in its coverage. The anchor stands by and replies by a beacon message to the sender. This message indicates the RSSI measurement and will be classified in a FLI classified in the set $\{$ Room_A, Room_B,...,Room_N\} (Fig. 4).

Thus, the fuzzy set "Room_A" is defined by Room_A $=\{$ FLI1, FLI2,.., FLI $n\}$. Hence, Room_B will be Room_B $=\{\operatorname{FLI} n+1, \operatorname{FLI} n+2, \ldots, \operatorname{FLI} n+m\}$ and so on for each room (Fig. 5). Those FSs are represented by type 1 triangular membership functions (Fig. 6).

The FLI specification will be equivalent to the FSs of Rssi vector in each room.

The variation in each FLI will specify the interval of each membership function. For $\mathrm{FLI}=1$, an interval of the lowest measured Rssi for FLI =1 and the higher measured one for the same FLI will be determined.

A subset Room $_{\mathrm{A}}$ of the set FLI is induced by its membership function $\mu_{\text {Room_A }}$ mapping the indicators of the FLI with the elements of the unity interval $[0,1]$, $\mu_{\text {Room_A }}:$ FLI $\rightarrow[0,1]$ (Fig. 6).

\subsection{FLS}

Considering the correlation between input fuzzy RSS sets and the output FLI, a Mamdani model is used, with the following characteristics:

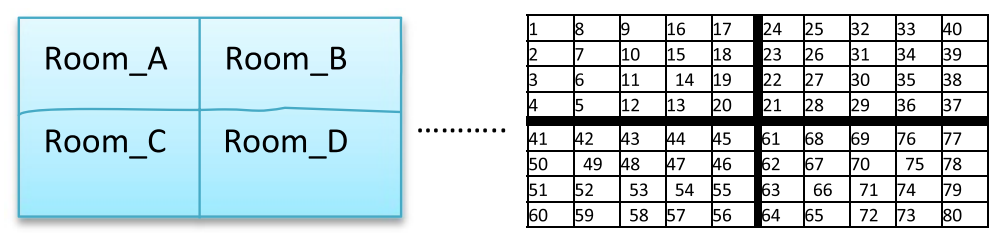

Fig. 5 Fuzzy location indicator (FLI) definition for room identification 


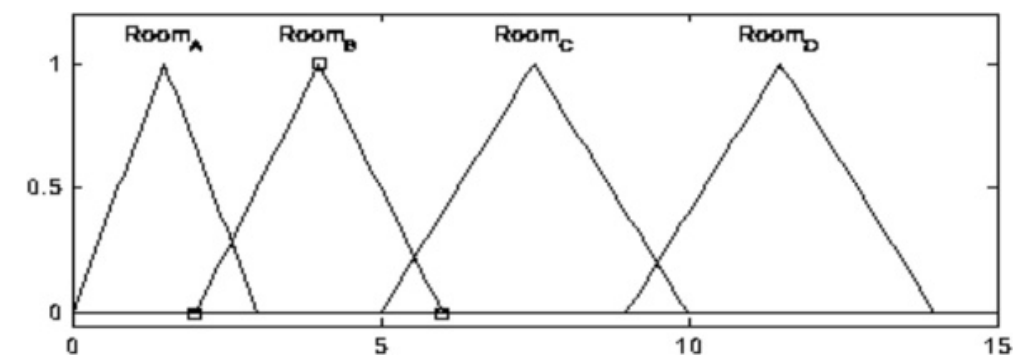

Fig. 6 Membership functions of the FLI

"Fuzzification = 'Interval Type 2"'

"Approach = 'Principal MF + FOU'"

"TNorm = "Product"

"SNorm = "Probor"

"Implication Method = 'Product'"

"Aggregation Method = 'Probor'"

"Type Reduction Method = 'LoM'"

"Defuzzification Method = 'Minimum"

Details of those methods may be found in [28] and [30].

\section{Experimental results and discussion}

\subsection{Experimental test bed}

For the experimental setup, we considered the implementation of a localization platform in the indoor environment of the Cynapsys Company (Fig. 7). The experimental bench is realized by the deployment of a WSN platform. The choice of the communication protocol and kit was restricted by the used sensors in Cynapsys for their smart home project. This platform is based on an STM32W108C kit set of four application boards to build a mesh network (two standard boards (MB851) and two with power amplifiers (MB954)). STM32W108 is a ZigBee RF4CE and IEEE 802.15.4 certified platform. The boards integrate a 2.4$\mathrm{GHz}$, IEEE802.15.4 compliant transceiver and a 32-bit $\mathrm{ARM}^{\circ}$-CortexTM M3 microprocessor. Thus, it allows evaluating IEEE 802.15.4 capabilities and developing a testing localization application.

Let us consider the plan of Cynapsys as our test bed. Three STM32W108 boards are deployed as anchors.
Figure 9 shows there dispositions in a way their range covers all the target zones.

The mobile node MB954 is attached to an i5 Pc through a USB cable. The expert takes five fingerprints in each indicated zone along the target space: \{Open_Space, Pythagore_meeting_room, Pythagore_corridor, Reception, Descartes_meeting_room, Descartes_corridor, RD_room\}.

The localization process is composed of two main phases. The first one is the "learning phase." In this stage, the expert saves the fingerprints relative to each room. In the second stage, the system proceeds to a fuzzy localization process.

\subsection{Software developments}

\subsubsection{Network creation}

The implementation of the localization platform was started by connecting the STM32W108 boards and building the network. In the first place, the choice of network topology was made based on the application needs. It is necessary to keep in obvious fact that the mobile board must be able to communicate directly with anchors so that we obtain the value of the RSSI between these two nodes and not with regard to another node which has broadcast the message. Based on the optimized MAC library IEEE 802.15.4, three possible topologies for the network are possible. The first one is star topology. In this type of network, all nodes are directly connected to the coordinator. Thus, there is no direct communication if we place more than one mobile. The second topology is in a tree where nodes which are
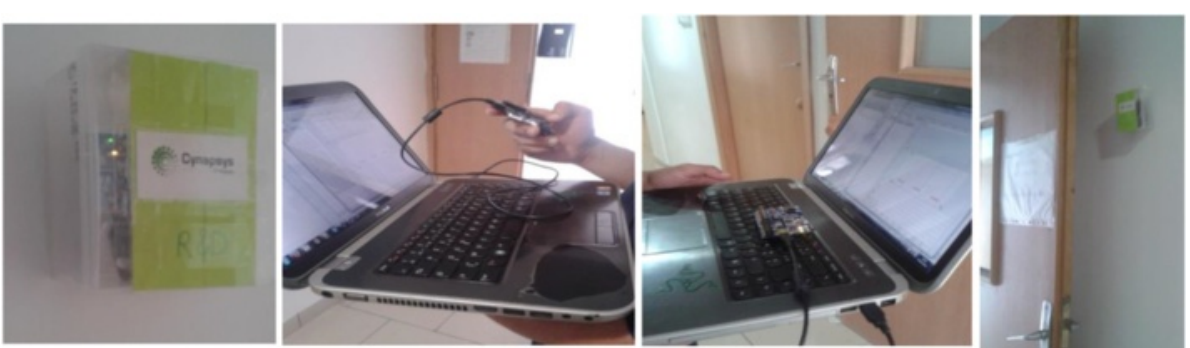

Fig. 7 Experimental setup of the localization test bed 


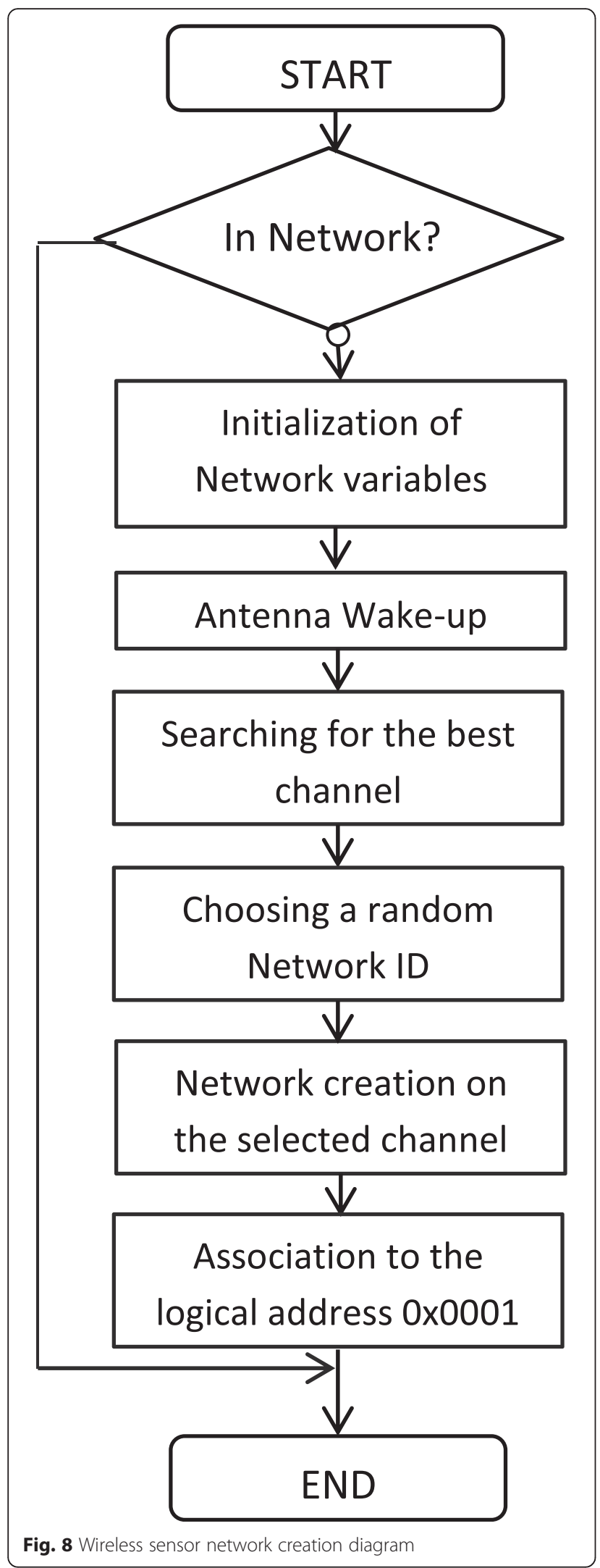

in various connections of the tree are not in direct contact what does not suit our application. The final proposed topology is meshed where nodes are in direct contact. This topology suits our case.

Hence, the embedded program is charged of RSSI collection and sending. The functions realized by the embedded system are the following ones: firstly, to establish a wireless network, then connecting cards on the same network; secondly, to assure a stable communication between them. In addition to making requests to collect RSSI from various anchors, it sends the RSSI vector collected and realizes commands reading via USB to manage card behavior.

The "Simple MAC" library is used for the program. One of its functions is the callback function named "ST_RadioReceiveIsrCallback" which is going to serve us to get back the value of the RSSI. The callback function types are generally functions of interruption which runs automatically in case of an event. In our case, the "ST_RadioReceiveIsrCallback" function runs in the reception of a message. It allows getting back the package in question, the time of arrival, if it contains errors, and the RSSI. Thanks to this function, the recovery of the RSSI of every message is possible; thus, it is enough to send any message to be able to know the RSSI with the transmitter.

To realize the communication between boards, it is necessary first of all to create a private network. The creation of the network is only realized by the coordinator which plays the role of a server. As indicated in Fig. 8, all at first begin by making out a choice, if the boards are already in a network or not. If not, the coordinator proceeds to the initialization of the network intern variables, and then, it wakes up the radio. Next, it looks for the best channel (energetic side) and it chooses randomly one ID for the network, and then, the latter is created in the chosen channel. Finally, it attributes the logical address 1 in the table of address. The protocol was developed in IAR Embedded Workbench for ARM 6.50 and loaded on the STM32W108 boards using a programmer and debugger JLink of flash memory JTAG.

\subsubsection{Input fuzzification}

As we considered in the localisation test bed of three anchors, three fuzzy inputs are defined: ZRSS1, ZRSS2, and ZRSS3. Their properties are as defined in Table 1 defined the same way for ZRSS2 and ZRSS3 on the Generalized Fuzzy System toolbox on Matlab R2014. The universe of discourse (UOD) was defined based on the experimental measurements, and FOU was defined as 2 .

\subsubsection{Output FLI definition}

The testing environment for localization on Cynapsys was divided into 20 zones. Each zone is defined by its 
Table 1 Fuzzy interval type 2 ZRSS1 properties

\begin{tabular}{|l|l|}
\hline ZRSS1 \\
UOD = [-100 0] \\
NumMFUNs = 3 \\
MF1 = 'Low':'Gaussian', [10-80],FOU = 2 \\
MF2 = 'Medium':'Gaussian',[10-50],FOU = \\
MF3 = 'High':'Gaussian',[10-20],FOU = 2
\end{tabular}

FLI, and every room is considered as a type 1 triangular fuzzy set presented in Fig. 9 from the left to the right and with the following parameters:

"MF1 = 'Open_Space':'Triangular',[0 1.5 3]"

"MF2 = 'Pythagore_room':'Triangular',[2.5 3.5 5]"

"MF3 = 'Pythagore_corridor':'Triangular',[4.5 6 8]"

"MF4 = 'Reception':'Triangular',[7 9 11]"

"MF5 = 'Descartes_room':'Triangular,'[10 12 14]"

"MF6 = 'Descartes_corridor':'Triangular,'[13 15 17]"

"MF7 = 'RD_room':'Triangular',[16 18 20]"

\subsubsection{Rule base creation}

Twenty-one rules were defined based on the expert linguistic evaluations. Some rules were technically impossible and were not considered, for example, IF (ZRSS1 IS Medium) (ZRSS2 IS High) (ZRSS3 IS High) is impossible, the mobile node cannot be near anchor 2 and anchor 3 in the same time.

1. IF (ZRSS1 IS Low) (ZRSS2 IS Low) (ZRSS3 IS Low)THEN (FLI IS Open_Space)(1)

2. IF (ZRSS1 IS Low) (ZRSS2 IS Low) (ZRSS3 IS Medium)THEN (FLI IS Open_Space)(1)

3. IF (ZRSS1 IS Low) (ZRSS2 IS Low) (ZRSS3 IS High)THEN (FLI IS Open_Space)(1)
4. IF (ZRSS1 IS Low) (ZRSS2 IS Medium) (ZRSS3 IS Low)THEN (FLI IS Open_Space)(1)

5. IF (ZRSS1 IS Low) (ZRSS2 IS High) (ZRSS3 IS Medium)THEN (FLI IS Open_Space)(1)

6. IF (ZRSS1 IS Medium) (ZRSS2 IS Low) (ZRSS3 IS Low)THEN (FLI IS Open_Space)(1)

7. IF (ZRSS1 IS Medium) (ZRSS2 IS Low) (ZRSS3 IS Medium)THEN (FLI IS Open_Space)(1)

8. IF (ZRSS1 IS Medium) (ZRSS2 IS Low) (ZRSS3 IS High)THEN (FLI IS Open_Space)(1)

9. IF (ZRSS1 IS Medium) (ZRSS2 IS Medium) (ZRSS3 IS Low)THEN (FLI IS Open_Space)(1)

10.IF (ZRSS1 IS Medium) (ZRSS2 IS Medium) (ZRSS3 IS Medium)THEN (FLI IS Open_Space)(1)

11.IF (ZRSS1 IS Low) (ZRSS2 IS Medium) (ZRSS3 IS Medium)THEN (FLI IS Open_Space)(1)

12.IF (ZRSS1 IS Low) (ZRSS2 IS Medium) (ZRSS3 IS High)THEN (FLI IS Open_Space)(1)

13.IF (ZRSS1 IS Low) (ZRSS2 IS High) (ZRSS3 IS Medium)THEN (FLI IS Open_Space)(1)

14.IF (ZRSS1 IS Medium) (ZRSS2 IS High) (ZRSS3 IS Low)THEN (FLI IS Open_Space)(1)

15.IF (ZRSS1 IS Medium) (ZRSS2 IS High) (ZRSS3 IS Medium)THEN (FLI IS Open_Space)(1)

16.IF (ZRSS1 IS High) (ZRSS2 IS Low) (ZRSS3 IS Low)THEN (FLI IS Open_Space)(1)

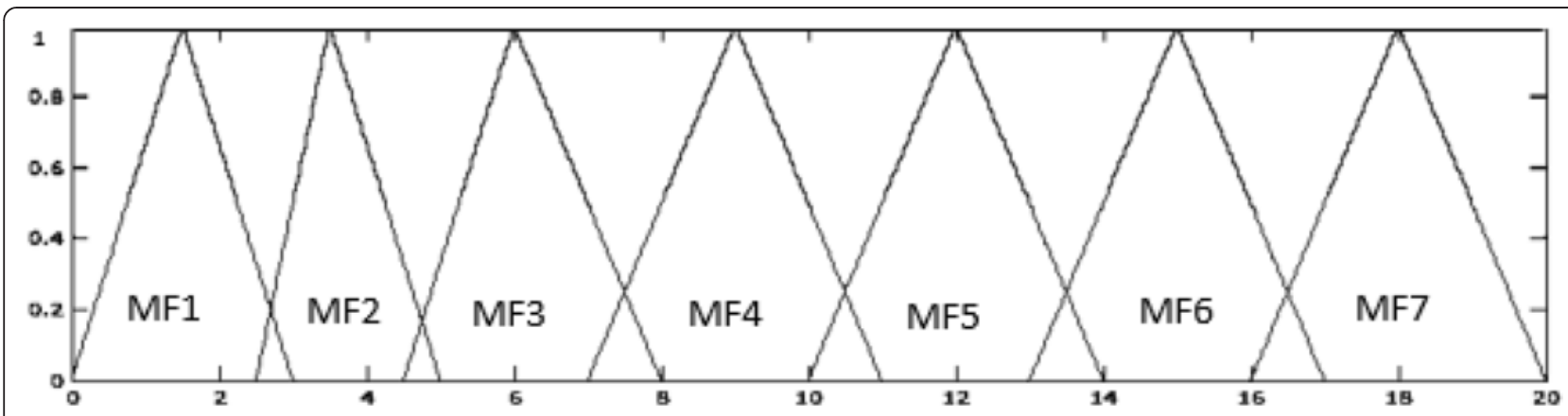

Fig. 9 FLI membership functions (MFs) 


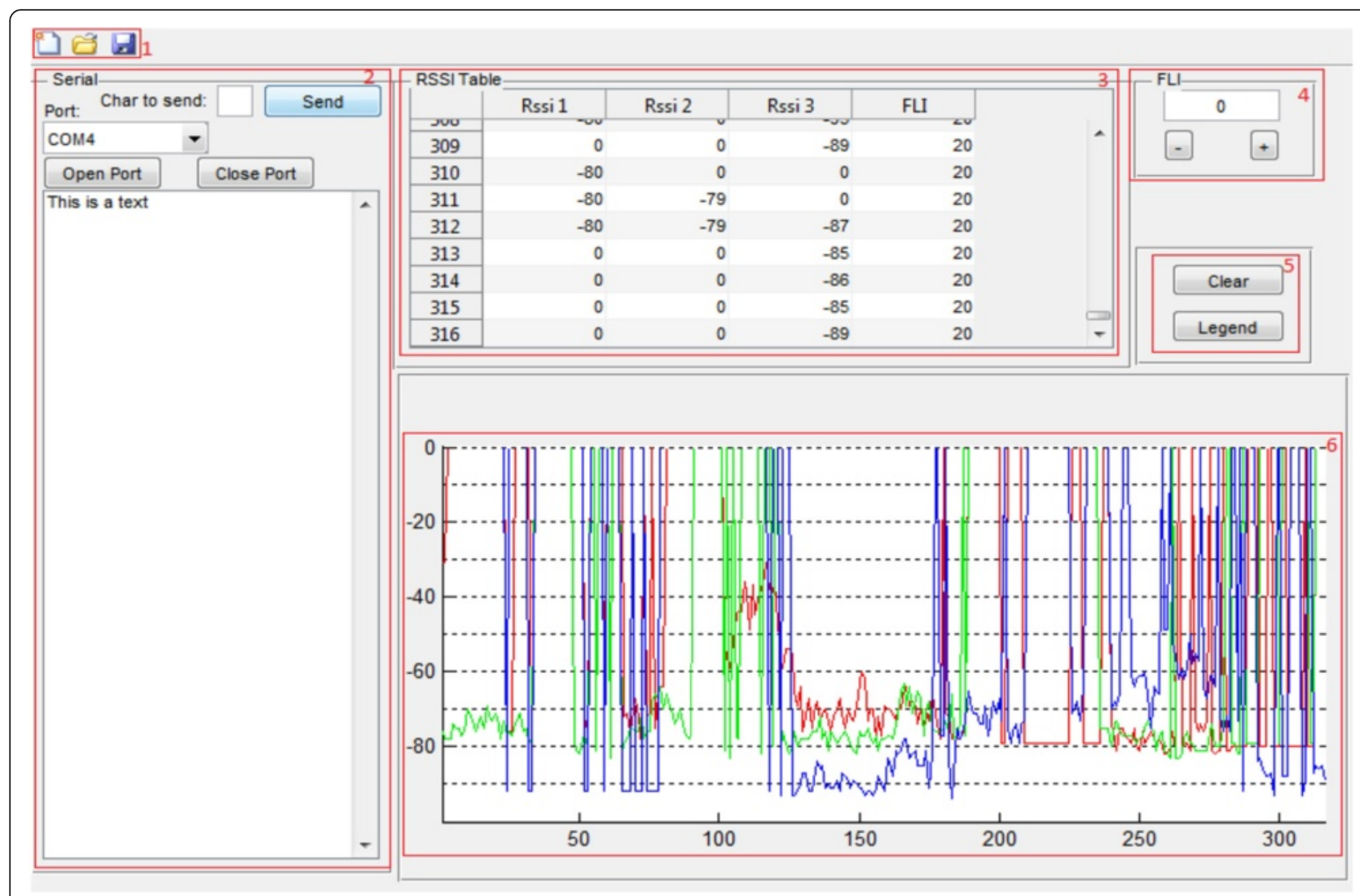

Fig. 10 Fingerprinting GUI

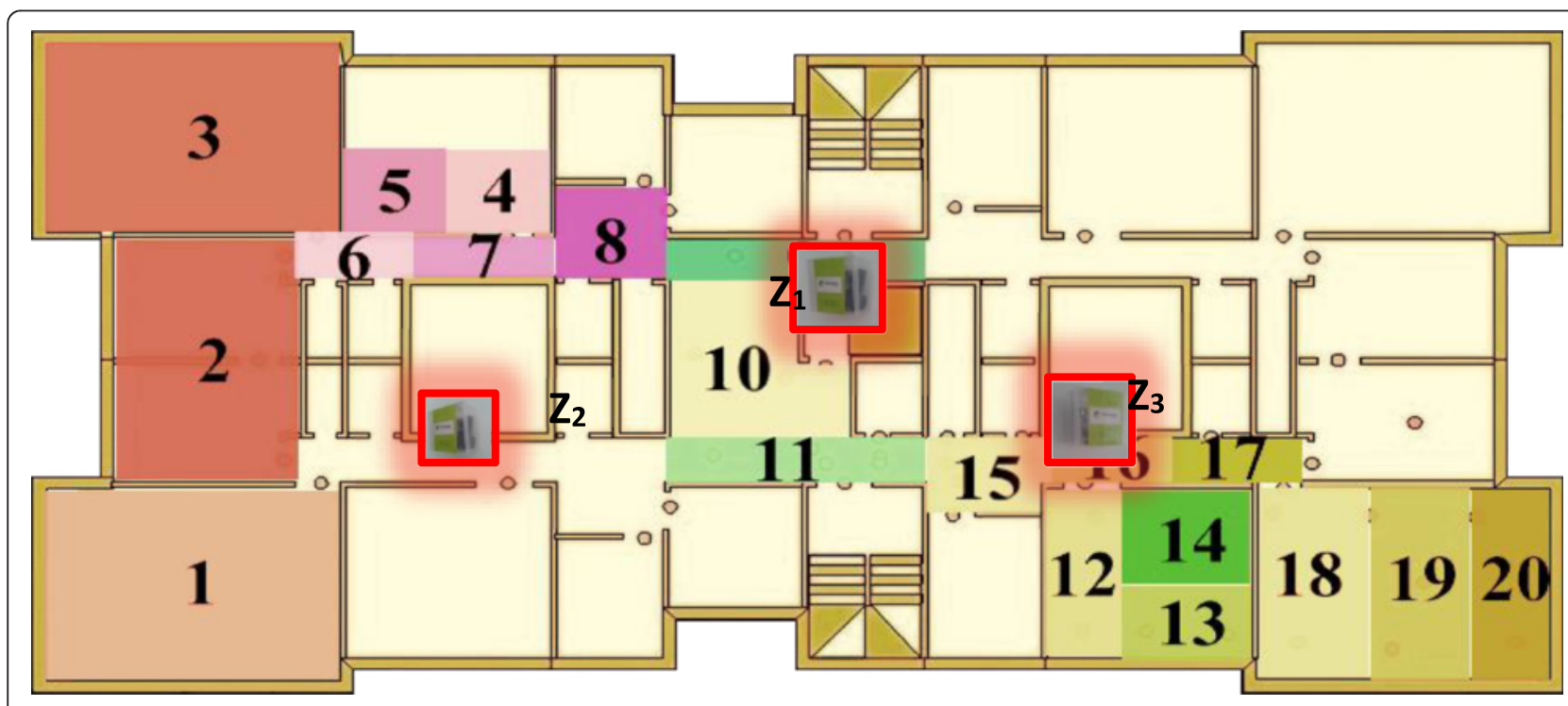

Fig. 11 Experimental setup of the localization bench 


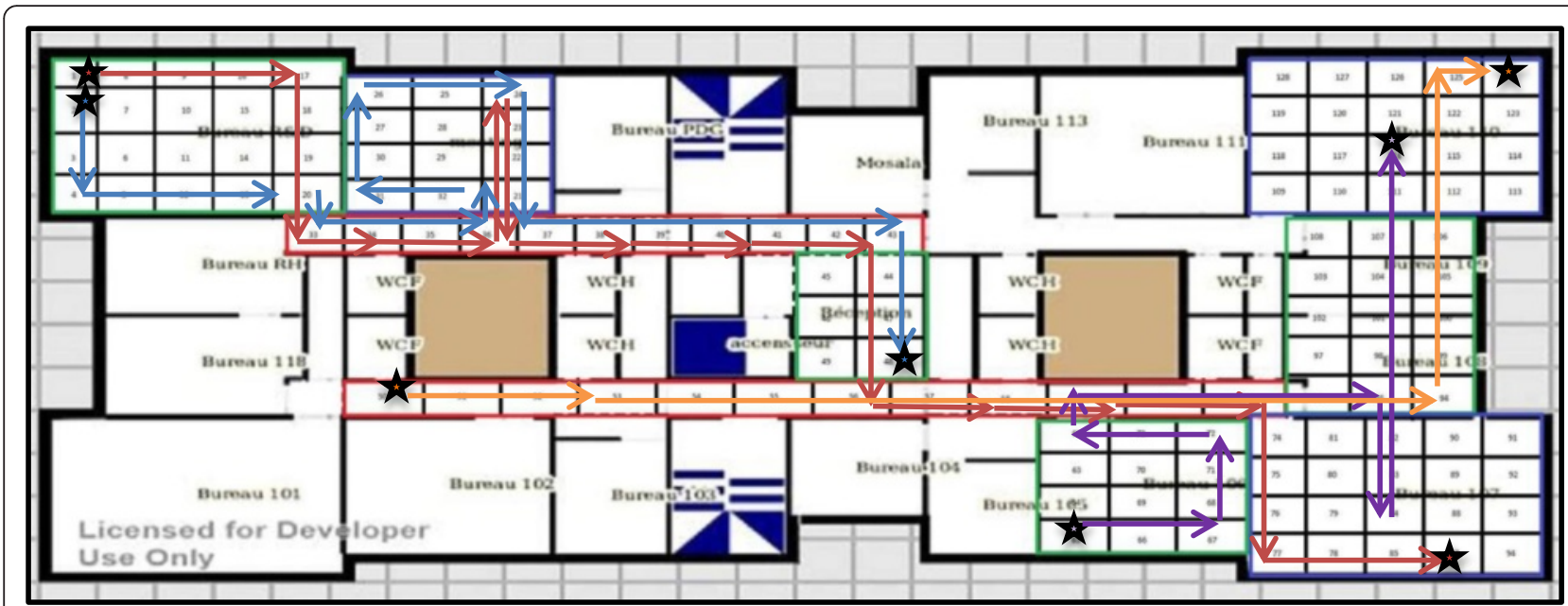

Fig. 12 Experimental scenarios on the Cynapsys map

17.IF (ZRSS1 IS High) (ZRSS2 IS Low) (ZRSS3 IS Medium)THEN (FLI IS Open_Space)(1)

18.IF (ZRSS1 IS High) (ZRSS2 IS Low) (ZRSS3 IS High)THEN (FLI IS Open_Space)(1)

19.IF (ZRSS1 IS High) (ZRSS2 IS Medium) (ZRSS3 IS Low)THEN (FLI IS Open_Space)(1)

20.IF (ZRSS1 IS High) (ZRSS2 IS Medium) (ZRSS3 IS Medium)THEN (FLI IS Open_Space)(1)

21.IF (ZRSS1 IS High) (ZRSS2 IS Medium) (ZRSS3 IS High)THEN (FLI IS Open_Space)(1)

\subsubsection{Experimental platform}

Using Matlab R2014, a fuzzy localization platform was developed. The graphical user interface (GUI) presents two windows. The first one, shown in Fig. 10, is dedicated to fingerprinting. In the second red rectangle of Fig. 10, the communicating interface between the anchor nodes and the mobile node linked to the PC through the "COM4" port.

The second interface processes those measurements and displays the calculated FLI on the map as presented in Fig. 11.

\subsection{Experimental scenarios}

Two test cases were defined. In the first test case, the target environment where divided into 20 zones as shown in Fig. 11. The average zone's area is $20 \mathrm{~m}^{2}$. In the second test case, we divided the target environment into 130 zones with an average area of $2.25 \mathrm{~m}^{2}$. For each test case, five scenarios were evaluated through five trajectories and four of them are shown in Fig. 12: blue, orange, and purple and the other trajectory repeats the fingerprinting trajectory; hence, it tested all the zones. For each trajectory, we started from the star point in a defined zone.

Then, relying on the RSSI measurements from the ZigBee anchors $Z_{1}, Z_{2}$, and $Z_{3}$, the system calculates the FLI, and using the algorithm below, the localization process is evaluated.

\subsection{Results and discussion}

While this work is based on a linguistic localization, the performance of the design is evaluated through the success and failure rate of the estimated position in the zone level and in the room level.

For the first test case where FLI $=20$, Table 2 details success rates in both zone and room levels. The true estimated position was mentioned by the user as correct during evaluation for two characteristics: is it the correct zone? And is it the correct room? Saved answers in the data base are used to calculate the success rate. From the fifth trajectories, less success rate was recorded for

Table 2 Experimental results for $\mathrm{FLI}=20$

\begin{tabular}{llllll}
\hline Scenarios & Total positions & True estimated position & True estimated room & $\begin{array}{l}\text { Success rate in } \\
\text { the zone level (\%) }\end{array}$ & $\begin{array}{l}\text { Success rate in the } \\
\text { room level (\%) }\end{array}$ \\
\hline Fingerprinting trajectory & 120 & 105 & 107 & 87.5 & 89.16 \\
Red trajectory & 65 & 57 & 57 & 87.69 & 87.69 \\
Orange trajectory & 18 & 16 & 16 & 88.88 & 88.88 \\
Purple trajectory & 30 & 24 & 25 & 80 & 83.33 \\
Blue trajectory & 30 & 25 & 25 & 83.33 & 83.33 \\
\hline
\end{tabular}


Table 3 Experimental results for $F L I=130$

\begin{tabular}{llllll}
\hline Scenarios & Total positions & True estimated position & True estimated room & $\begin{array}{l}\text { Success rate in } \\
\text { the zone level (\%) }\end{array}$ & $\begin{array}{l}\text { Success rate in } \\
\text { the room level (\%) }\end{array}$ \\
\hline Fingerprinting trajectory & 120 & 98 & 110 & 81.66 & 91.66 \\
Red trajectory & 65 & 55 & 59 & 84.61 & 90.76 \\
Orange trajectory & 18 & 16 & 16 & 88.88 & 88.88 \\
Purple trajectory & 30 & 24 & 28 & 80 & 93.33 \\
Blue trajectory & 30 & 22 & 25 & 73.33 & 83.33 \\
\hline
\end{tabular}

the longer trajectory where all zones are crossed. Errors in the zone level are not necessarily errors in the room level. In the purple trajectory, for example, six errors were noticed. One error was a wrong zone but in the correct room, and the others are wrong zones and wrong room estimation. Higher success rate was recorded on the orange trajectory positions. This is due to the fact that this trajectory presents a simple path where less neighboring rooms and zones cause similarity. Hence, the average success rate in the zone level is $85.48 \%$, and the average success rate for the room level is $86.47 \%$ which is an excellent positioning rate.

For the second test case where FLI $=130$, harder work was done to collect five fingerprints in a $2.25 \mathrm{~m}^{2}$ zone. Their experimental results presented in Table 3 shows an average success rate in the zone level as $81.69 \%$ and an average success rate in the room level as $89.59 \%$. In comparison to the first case, the success rate in the zone level was decreased but the success in the room level was increased. This is because the number of zones in a room rises, for example for RD room, it was only 3 zones and became 20 zones. Hence, the positioning errors in the zones become more frequent. Besides, errors when taking fingerprintings have higher probability to occur while zones are small $\left(2.25 \mathrm{~m}^{2}\right)$ and fingerprints in the same room are nearly similar.
On the other side, the localization system was tested using a T1 RSS signal processing. For FLI $=20$, the average success rate has remarkably decreased in the zone level to $75.59 \%$ and the average success rate for the room level to $77.4 \%$. For $F L I=130$, it decreases to $72.39 \%$ in the zone level and to $78.88 \%$ in the room level. Hence, the use of IT2 fuzzy logic has enhanced the performance of the system by $10 \%$ as presented in Fig. 13, despite that the FLI approach has proved good localization precision regards to fuzzy systems.

Further experiments were conducted on the R\&D room $(8 \mathrm{~m} \times 5.5 \mathrm{~m})$ to calculate the average localization error (The localization error is measured as the Euclidean distance between the actual and the estimated locations). For three anchor boards (STM32W108), 200 fingerprints were taken through $40 \mathrm{FLI}$ in the target zone. Thirty-five locations were considered for evaluation. For the sake of consistency and completeness, we use our gathered data to evaluate our proposed IT2FL algorithm and two nonfuzzy algorithms: the KNN-based localization method and the lateration algorithm.

The KNN method is based on comparative searches of the profiled fingerprints to choose the $\mathrm{K}$ closest profiled samples in terms of minimizing the RSS discordance between the query RSS sample and the profiled ones.

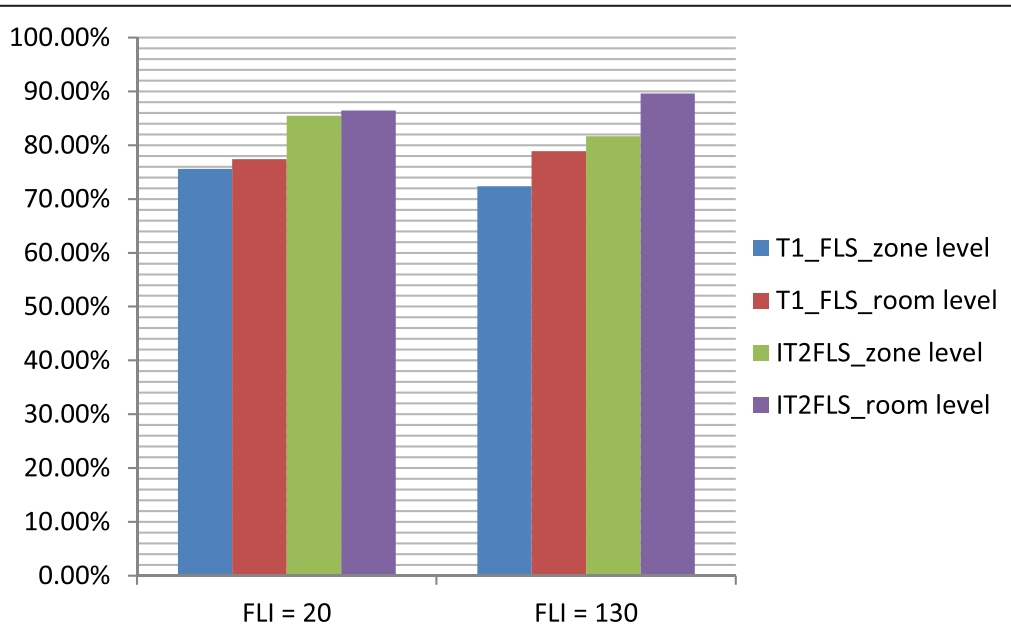

Fig. 13 Success rate in $\mathrm{T} 1$ and IT2FLS for FLI = 20 and 130 
Table 4 Comparative experimental results of the average localization error between fuzzy and non-fuzzy algorithms

\begin{tabular}{lc}
\hline Localization algorithm & Average localization error $(\mathrm{m})$ \\
\hline IT2FLS & 0.8 \\
K-nearest neighbor (KNN) & 1.1 \\
Lateration & 1.9
\end{tabular}

The weighted coordinates of these $K$ samples generate the estimated location.

For the lateration-based localization algorithm, a lognormal shadowing propagation model is configured as follows:

$$
\operatorname{RSS}(i)=\mathrm{RSS} 0-10 * \mathrm{np} * \log (d(i) / d 0)+\sigma
$$

where RSS0 parameter refers to the initial signal strength in $d 0$ (for our experimental bed RSS0 $=-63 \mathrm{dBm}$, for $d 0=$ $1 \mathrm{~m}$ ), $\mathrm{np}$ is the path loss parameter $\mathrm{np}=2.25$ (considering an indoor office environment), and $\sigma$ is the standard deviation of valid RSSI values per link. We use the profiled fingerprints to estimate $\sigma=4.9 \mathrm{~dB}$. Then, the system of equation system is solved using a standard least squares approach [31] deducing the average error distance.

Table 4 exhibits the average localization error of the testing fingerprinting sets. The experimental results of our proposed approach outperform the two non-fuzzy localization-based algorithms followed by the KNN-based and then by the lateration method. The integration of interval type 2 fuzzification has limited the effect of RSSi fluctuations. Hence, the proposed localization system performs better regarding the obstacle's presence in indoor environments. It integrates a linguistic classification approach, simplifying the KNN-based classifications and reducing the heavy calculations found in the lateration method.

\section{Conclusions}

This paper proposed a linguistic fuzzy modeling focused on interpretability for localization of mobile nodes in wireless sensor networks. The uncertainty in the linguistic localization system was processed in two ways: in the first place, an interval type 2 fuzzification was proposed to handle RSSI fluctuations. Secondly, a fuzzy location indicator (FLI) was considered to handle geometric repartitions of fuzzy fingerprints. Experimentations have proved a high success rate either for the zone level or the room level. Besides, the superiority of IT2FL to T1FL to minimize RSSI uncertainties has been proved.

In the future work, we intend to work on the automatic generation of the rule base through a neuro-fuzzy algorithm (GARIC), in addition to automating the FLI recording through a preprogrammed drone.

\section{Competing interests}

The authors declare that they have no competing interests.

\section{Acknowledgements}

These works of research and innovation are made within a MOBIDOC thesis, financed by the European Union (EU) within the framework of the PASRI program, and partially supported by Cynapsys IT Enterprise. We give our thanks to Mootez Jridi and Meher Houidi for their assistances.

\section{Author details}

'Innov'COM/ENIT/Cynapsys, University Tunis El Manar, Tunis, Tunisia.

${ }^{2}$ Innov'COM, University Carthage, Tunis, Tunisia.

Received: 10 August 2015 Accepted: 23 March 2016

Published online: 02 April 2016

\section{References}

1. R Martinez, O Castillo, LT Aguilar, Optimization of interval type-2 fuzzy logic controllers for a perturbed autonomous wheeled mobile robot using genetic algorithms. Inf Sci 179(13), 2158-2174 (2009)

2. $\mathrm{C}-\mathrm{H}$ Hsu, C-F Juang, Evolutionary robot wall-following control using type-2 fuzzy controller with species-DE-activated continuous ACO. IEEE Trans. Fuzzy Syst. 21(1), 100-112 (2013)

3. R John, P Innocent, M Barnes, Type 2 fuzzy sets and neuro-fuzzy clustering of radiographic tibia images. Proceedings of the Sixth IEEE International Conference in Fuzzy Systems 3, 1375-1380 (1997)

4. H Hagras, Type-2 FLCs: a new generation of fuzzy controllers. IEEE Comput. Intell. Mag. 2(1), 30-43 (2007)

5. A Rubio Solis, G Panoutsos, Interval type-2 radial basis function neural network: a modelling framework. IEEE Trans. Fuzzy. Syst. 23(2), 457-473 (2015)

6. R Sepúlveda, O Castillo, P Melin, A Diaz, O Montiel, Handling Uncertainty in Controllers Using Type-2 Fuzzy Logic. Proceeding of the 2005 IEEE International Conference on Fuzzy Systems,USA, 2005, pp. 248-253

7. J Mendel, Type-2 fuzzy sets and systems: an overview. IEEE Comput. Intell. Mag. 2(2), 20-29 (2007)

8. $\quad$ ABI . Research, "Indoor location technologies," US, AN-1331 https://www. abiresearch.com/market-research/product/1016891-indoor-locationtechnology-oems/. (2014)

9. P Steinhaus, M Strand, R Dillmann, Autonomous robot navigation in human-centered environments based on 3D data fusion. EURASIP J. Adv. Signal Process. 2007(86831), 1-10 (2006)

10. H Liu, H Darabi, P Banerjee, J Liu, Survey of wireless indoor positioning techniques and systems. IEEE T. Syst. Man CY. C. 37(6), 1067-1080 (2007)

11. J Yan, K Yu, L Wu, Fuzzy modeling, maximum likelihood estimation, and Kalman filtering for target tracking in NLOS scenarios. EURASIP J. Adv. Signal Process. 2014, pp. 1-16 (2014)

12. M.R. Morelande, B. Moran, M. Brazil, "Bayesian node localisation in wireless sensor networks," in Acoustics, Speech and Signal Processing. ICASSP 2008. IEEE International Conference on, Las Vegas pp. 2545-2548, (2008)

13. H. Ahmadi and R. Bouallegue, "Comparative study of learning-based localization algorithms for Wireless Sensor Networks: Support Vector regression, Neural Network and Naïve Bayes," Wireless Communications and Mobile Computing Conference (IWCMC) proceedings, Dubrovnik, pp. 15541558, 2015.

14. IT Haque, C Assi, Profiling-based indoor localization schemes. IEEE Syst. J. 9(1), 76-85 (2015)

15. MA Alsheikh, S Lin, D Niyato, H-P Tan, Machine learning in wireless sensor networks: algorithms, strategies, and applications. IEEE Commun. Surv. Tutorials 16(4), 1996-2018 (2014)

16. A Ukil, Support Vector Machine, Chapter 4, Intelligent Systems and Signal Processing in Power Engineering (Springer-Verlag, Berlin, 2007)

17. L Gogolak, S Pletl, D Kukolj, Neural network-based indoor localization in WSN environments. Acta. Polytechnica. Hungarica. 10(6), 221-235 (2013)

18. P. Bahl and V. N. Padmanabhan, "RADAR: an in-building RF-based user location and tracking system," in INFOCOM 2000. Nineteenth Annual Joint Conference of the IEEE Computer and Communications Societies. Proceedings. IEEE pp 775-784 vol.2, Tel Aviv (2000). http://ieeexplore.ieee.org/xpl/ articleDetails.jsp?arnumber $=832252 \&$ newsearch=true\&queryText=RADAR: \%20an\%20in-building\%20RFbased\% 20user\%20location\%20and\%20tracking\%20system 
19. T. Oktem, D. Slock, "Pairwise error probability analysis for power delay profile fingerprinting based localization, pp. 1-5.," in Vehicular Technology Conference (VTC Spring), 2011 IEEE 73rd, Yokohama (2011). http://ieeexplore. ieee.org $/ \mathrm{xpl} /$ articleDetails.jsp?reload=true\&arnumber $=5956780$

20. R. Wang, W. Cao, W. Wan, Location discovery based on fuzzy geometry in passive sensor networks. Int Journal of Digital Multimedia Broadcasting. , vol. 2011, Article ID 851951, 6 pages. doi:10.1155/2011/851951 Hindawi Pub. Co, (2011)

21. E Fan, W-x Xie, Z-x Liu, Maneuvering target tracking using fuzzy logicbased recursive least squares filter. EURASIP J. Adv. Signal Process. 53(53), 1-16 (2014)

22. T Garcia-Valverde, A Garcia-Sola, A Gomez-Skarmeta, J Botia, H Hagras, J Dooley, $\checkmark$ Callaghan, "An adaptive learning fuzzy logic system for indoor localisation using Wi-Fi in ambient intelligent environments". Proc. FUZZ-IEEE 1(8), Brisbane, pp 10-15 (2012)

23. T Garcia-Valverde, A Garcia-Sola, H Hagras, J Dooley, V Callaghan, J Botia, A fuzzy logic-based system for indoor localization using WiFi in ambient intelligent environments. IEEE Trans. Fuzzy Systems 21(4), 702-718 (2013)

24. A Velimirovic, G Lj, M Djordjevic, M Velimirovic, M Jovanovic, A fuzzy set based approach to range-free localization in wireless sensor networks. Ser.:Elec.Energ. 23(2), 227-244 (2010)

25. A. Salazar, A. L and G. Licea, "Estimating indoor zone-level location using Wi-Fi RSSI fingerprinting based on fuzzy inference system," ICMEAE, Conf proceedings, Morelos pp. 19-22, 2013.

26. Q. Liang and L. Wang, "Sensed Signal Strength Forecasting for Wireless Sensors Using Interval Type-2 Fuzzy Logic System," in The 14th IEEE International Conference on Fuzzy Systems (FUZZ '05) pp. 25-30., 2005.

27. S. Satvir, S. G. Inderjeet, S. Sarabjeet and D. Gaurav, "Application of type-2 fuzzy logic-a review," International Conference on Communication, Computing \& Systems (ICCCS) proceedings, Punjab pp. 259-263, 2014.

28. J. Aladi, C. Wagner, J. Garibaldi, "Type-1 or interval type-2 fuzzy logic systems - on the relationship of the amount of uncertainty and FOU size," Fuzzy Systems (FUZZ-IEEE), 2014 IEEE International Conference on, Beijing pp. 2360-2367, 2014.

29. A. Sadeghian, J. Mendel, H. Tahayori, "Advances in type-2 fuzzy sets: theory and applications", Springer. Vol 30 (2013)

30. JM Mendel, Uncertain Rule-Based Fuzzy Logic Systems Introduction and New Directions (Prentice Hall, Indiana , 2001). http://www.informit.com/store/ uncertain-rule-based-fuzzy-logic-systems-introduction-9780130409690.

31. K Langendoen, N Reijers, Distributed localization in wireless sensor networks: a quantitative comparison. J. Comput. Netw. 43(3), 499-518 (2003). http://dx.doi.org/10.1016/S1389-1286(03)00356-6

\section{Submit your manuscript to a SpringerOpen ${ }^{\circ}$ journal and benefit from:}

- Convenient online submission

- Rigorous peer review

- Immediate publication on acceptance

- Open access: articles freely available online

- High visibility within the field

- Retaining the copyright to your article 